\title{
Peertechz
}
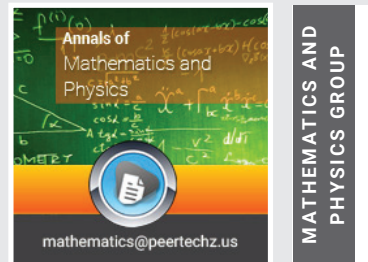

\section{On the special spherical triangles for physical and cosmological applications}

\author{
S Kalimuthu* \\ 2/394, Kanjampatti P.O, Pollachi Via, Tamil Nadu 642003, India
}

\begin{abstract}
Received: 14 July, 2021
Accepted: 24 November, 2021

Published: 25 November, 2021

*Corresponding authors: S Kalimuthu, 2/394, Kanjampatti P.O, Pollachi Via, Tamil Nadu 642003, India, Tel: + 918220541577

E-mail: owlskalimuthu@gmail.com

Copyright: (c) 2021 Kalimuthu S. This is an openaccess article distributed under the terms of the Creative Commons Attribution License, which permits unrestricted use, distribution, and reproduction in any medium, provided the original author and source are credited.
\end{abstract}

https://www.peertechzpublications.com

\section{Check for updates}

It is well known that a spherical triangle of 270 degree triangle is constructible on the surface of a sphere; a globe is a good example. Take a point (A) on the equator, draw a line $1 / 4$ the way around (90 degrees of longitude) on the equator to a new point (B).... The angle at each of the vertices $(A, B, C)$ will be ninety degrees, for a total of 270 degrees as shown in Figure 1. It is also possible to draw a spherical triangle whose interior angle sum is equal to 360 degrees. Also, it is possible to construct a special spherical triangle whose interior angle sum up to 540 degrees.

The physical and cosmological applications of the similar triangles of these three special spherical triangles are discussed in this work

MSC : 51M09, 51M10, 51M15, 51P05

PACS : 02.40.Ky

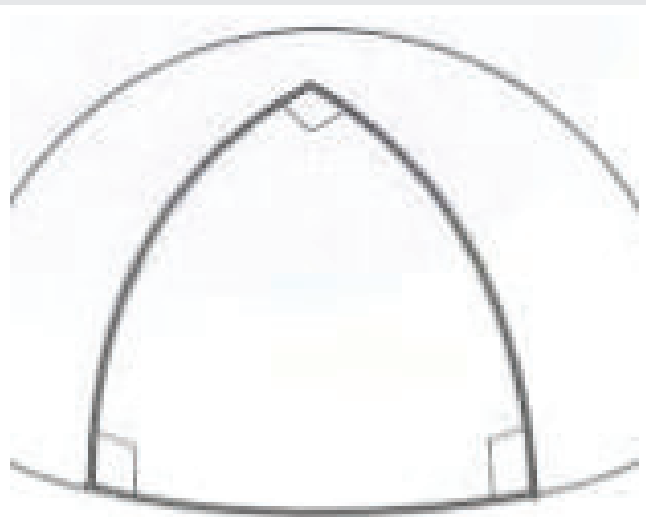

Figure 1: Spherical Triangle.
Theorem 1 : There exists a spherical triangle whose interior angle sum adds to 360 degrees

Theorem 2 : There exists a spherical triangle whose interior angle sum adds to 540 degrees

\section{Construction}

Proof of theorem 1: In spherical Figure 2. Consider NB , WE and EN as the three sides of triangle NEW. WE is the equator of spherical Figure 1 and both EN and WN are perpendiculars to WE. Since the angle WNE is a straight angle, we get that the sum of the interior angles of spherical triangle WNE is equal to 360 degrees. And hence the proof.

Proof of theorem 2: In spherical Figure 3, consider WN , NE and EW as the three sides of spherical triangle WNE. Since the angles WNE , NEW and EWN are straight angles, we obtain that the sum of the interior angles of spherical triangle WNE is 540 degrees. And hence the proof.

\section{Discussion}

\section{On similar triangles in euclidean geometry}

In the classical Euclidean triangles, the following similar triangles phenomena are hold good:

\section{Theorem 1}

If one triangle has a pair of angles that are congruent to a pair of angles in another triangle, then the two triangles are similar.

\section{Theorem 2}

If a pair of sides of a triangle is proportional to a pair of 

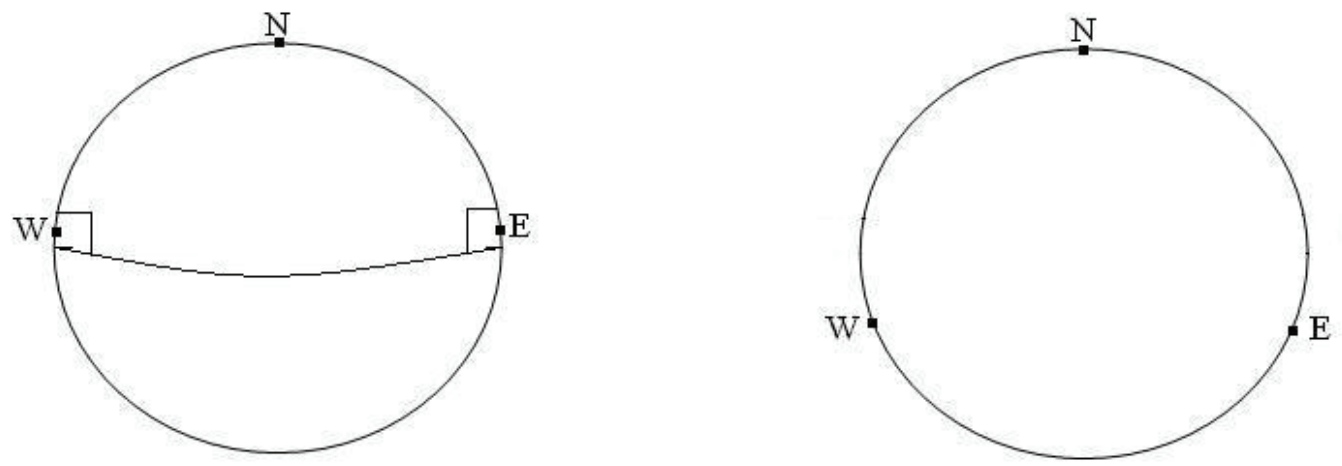

Figures 2,3: Spherical.

sides in another triangle and if the angles included by the sidepairs are congruent, then the triangles are similar.

\section{Theorem 3}

If the sides of a triangle are proportional to the sides of another triangle, then the triangles are similar.

Let us recall the following columns in:https://mathstat.slu. edu/escher/index.php/The_Three_Geometries

\section{On similar triangles in hyperbolic geometry}

"In hyperbolic geometry, a hyperbolic triangle is a triangle in the hyperbolic plane. It consists of three line segments called sides or edges and three points called angles or vertices. Just as in the Euclidean case, three points of a hyperbolic space of an arbitrary dimension always lie on the same plane.

There are no similar triangles in hyperbolic geometry: The best-known example of a hyperbolic space are spheres in Lorentzian four-space.

Similar triangles do not exist in the Hyperbolic Geometry. That is because exists a relation between angles and distances given by the angle of parallelism formula. The non-existence of similar hyperbolic triangles implies the existence of a unique hyperbolic equilateral triangle fixed the angle.

As it is known, in Hyperbolic Geometry, the sum of the angles of a triangle is always less than two right angles. So, each angle of an equilateral triangle will not have exactly $60^{\circ}$. It can be seen that for any value among 0 and $60^{\circ}$, both non included, we have an equilateral triangle, which is unique, except isometries.

\section{On similar triangles in spherical geometry}

The angle between two lines in spherical geometry is the angle between the planes of the corresponding great circles, and a spherical triangle is defined by its three angles. ... There is no concept of similar triangles in spherical geometry.

It has been thought that there are no similar triangles on the sphere, but in fact they are not. There are also similar triangles on the sphere, the similar conditions are: the corresponding sides are parallel and proportional, and the corresponding angles are equal. The similarity on a sphere is not exactly the same as that on a plane. However, the similarity on a plane is a special case of the similarity on a sphere. am I correct?

Triangles whose sides are formed by great arcs are triangles. In my definition, triangles whose sides are formed by arcs are also triangles. My definition is more general.

In addition, there are similar squares on the sphere. It should be noted that the sum of the inner angles of the squares on the sphere is greater than $2 \pi$.

\section{The five axioms for euclidean geometry are}

1. Any two points can be joined by a straight line. (This line is unique given that the points are distinct)

2. Any straight line segment can be extended indefinitely in a straight line.

3. Given any straight line segment, a circle can be drawn having the segment as radius and one endpoint as center.

4. All right angles are congruent.

5. Through a point not on a given straight line, one and only one line can be drawn that never meets the given line.

\section{The five axioms for hyperbolic geometry are}

1. Any two points can be joined by a straight line.

2. Any straight line segment can be extended indefinitely in a straight line.

3. Given any straight line segment, a circle can be drawn having the segment as radius and one endpoint as center.

4. All right angles are congruent.

5. Through a point not on a given straight line, infinitely many lines can be drawn that never meet the given line. 


\section{How do we interpret the first four axioms on the sphere?}

Lines: What would a "line" be on the sphere? In Euclidean geometry a line segment measures the shortest distance between two points. This is the characteristic we want to carry over to spherical geometry. The shortest distance between two points on a sphere always lies on a great circle. Longitude lines and the equator on a globe are examples of great circles. Note that we can always draw a great circle, which we will from now on call a geodesic, through any two points. We have to be careful here, because unlike in Euclidean geometry this geodesic ("line") may not be unique. Take for instance the north and South Pole on the globe. There are infinitely many great circles through these two poles. In general, any two points that lie on opposite sides of the sphere, so called antipodal points, can be joined by infinitely many geodesics.

Line segments: We can extend any line segment, but at some point the line segment will then connect up with itself. A line of infinite length would go around the sphere an infinite amount of times.

Circles: As we have stated the circle axiom it is true on the sphere. Note that it does not make sense to say that given any center $\mathrm{C}$ and any radius $\mathrm{R}$ we can draw a circle of radius $R$ centered at $C$. If we take a radius less than half the circumference of the sphere, then we can draw the circle. If the radius is exactly half the circumference of the sphere, then the circle degenerates into a point. If the radius were greater than half the circumference of the sphere, then we would repeat one of the circles described before. Note that great circles are both geodesics ("lines") and circles.

Angles: Right angles are congruent. Think about the intersection of the equator with any longitude. These two geodesics will meet at a right angle.

\section{How can we formulate the $5^{\text {th }}$ postulate?}

No parallel lines: Any two geodesics will intersect in exactly two points. Note that the two intersection points will always be antipodal points.

Sum of the angles in a triangle: On the sphere the sum of the angles in a triangle is always strictly greater than 180 degrees.

\section{The five axioms for spherical geometry are}

1. Any two points can be joined by a straight line.

2. Any straight line segment can be extended indefinitely in a straight line.

3. Given any straight line segment, a circle can be drawn having the segment as radius and one endpoint as center.

4. All right angles are congruent.

5. There are NO parallel lines.

\section{How do we interpret the first four axioms on the sphere?}

Lines: What would a "line" be on the sphere? In Euclidean geometry a line segment measures the shortest distance between two points. This is the characteristic we want to carry over to spherical geometry. The shortest distance between two points on a sphere always lies on a great circle. Longitude lines and the equator on a globe are examples of great circles. Note that we can always draw a great circle, which we will from now on call a geodesic, through any two points. We have to be careful here, because unlike in Euclidean geometry this geodesic ("line") may not be unique. Take for instance the north and South Pole on the globe. There are infinitely many great circles through these two poles. In general, any two points that lie on opposite sides of the sphere, so called antipodal points, can be joined by infinitely many geodesics.

Line segments: We can extend any line segment, but at some point the line segment will then connect up with itself. A line of infinite length would go around the sphere an infinite amount of times.

Circles: As we have stated the circle axiom it is true on the sphere. Note that it does not make sense to say that given any center $\mathrm{C}$ and any radius $\mathrm{R}$ we can draw a circle of radius $R$ centered at $C$. If we take a radius less than half the circumference of the sphere, then we can draw the circle. If the radius is exactly half the circumference of the sphere, then the circle degenerates into a point. If the radius were greater than half the circumference of the sphere, then we would repeat one of the circles described before. Note that great circles are both geodesics ("lines") and circles.

Angles: Right angles are congruent. Think about the intersection of the equator with any longitude. These two geodesics will meet at a right angle [1,2]

\section{Conclusion}

Let us assume that the three types of special spherical triangles discussed above is on a sphere $\mathrm{S}_{1}$ Let us draw such triangles in sphere $\mathrm{S}_{2}$ and sphere $\mathrm{S}_{3}$ Consequently, we obtain that these spherical triangles are similar. The author appeals to the research community to probe into this finding for future application in physics and cosmology.

In hyperbolic geometry the author politely introduces the following concept. Let $\mathrm{H}_{1}$. is the given hyperbolic triangle. Magnify this triangle and let it be $\mathrm{H}_{2}$ It is well known that in magnification, the angels are preserved. So, these hyperbolic triangles $\mathrm{H}_{1}$ and $\mathrm{H}_{2}$ are similar. The author appeals to the research community to delve and to probe in to this so that new clues may be explored.

\section{References}

1. The Three Geometries. Link: https://bit.ly/3DMZSf6

2. Link: https://bit.ly/3xkKzYl 